\title{
Intercavernous Sinus Dural Arteriovenous Fistula Treated with Transvenous Coil Embolization: A Case Report
}

\author{
Mostafa Saleh Melake ${ }^{a}$ Hidenori Oishi $^{b}$ Yasuo Suga ${ }^{b}$ \\ Munetaka Yamamoto ${ }^{b}$ Hajime Arai ${ }^{b}$ \\ a Juntendo School of Medicine, and ' ${ }^{b}$ Department of Neuroendovascular Surgery, \\ Juntendo School of Medicine, Tokyo, Japan
}

\section{Key Words}

Dural arteriovenous fistula $\cdot$ Intercavernous sinus $\cdot$ Coil embolization

\begin{abstract}
The blood supply to the region of the cavernous sinus is provided by interconnecting branches of the internal and external carotid arteries, and it is from these vessels that dural arteriovenous fistulas (DAVFs) arise. It is very rare that DAVFs are located at the intercavernous sinuses region; in this case, a proper localization of the fistulous site is of extreme importance in order to successfully eliminate the disease. Here, we describe a case of a 65-year-old female with intercavernous sinus DAVF. A complete obliteration of the fistulous points was performed by coil embolization through a transvenous approach.

(C) 2014 S. Karger AG, Basel
\end{abstract}

\section{Introduction}

The blood supply to the region of the cavernous sinus is provided by interconnecting branches of the internal and external carotid arteries, and it is from these vessels that dural carotid cavernous fistulas - often called dural arteriovenous fistulas (DAVFs) - arise. Such fistulas usually are separated anatomically into three types: (1) shunts between meningeal branches of the internal carotid artery and the cavernous sinus; (2) shunts between meningeal branches of the external carotid artery and the cavernous sinus, and (3) shunts between meningeal branches of both the internal and external carotid arteries and the cavernous sinus. Of these types, the third is by far the most common [1]. Cavernous sinus DAVFs can 
Fig. 1. Image of the patient showing bilateral congested eyes, conjuctival edema, and right abducens palsy.

\begin{tabular}{l|l}
\hline DOI: $10.1159 / 000365554$ & $\begin{array}{l}\text { C 2014 S. Karger AG, Basel } \\
\text { www.karger.com/ine }\end{array}$ \\
\hline
\end{tabular}

Melake et al.: Intercavernous Sinus Dural Arteriovenous Fistula Treated with Transvenous Coil Embolization: A Case Report

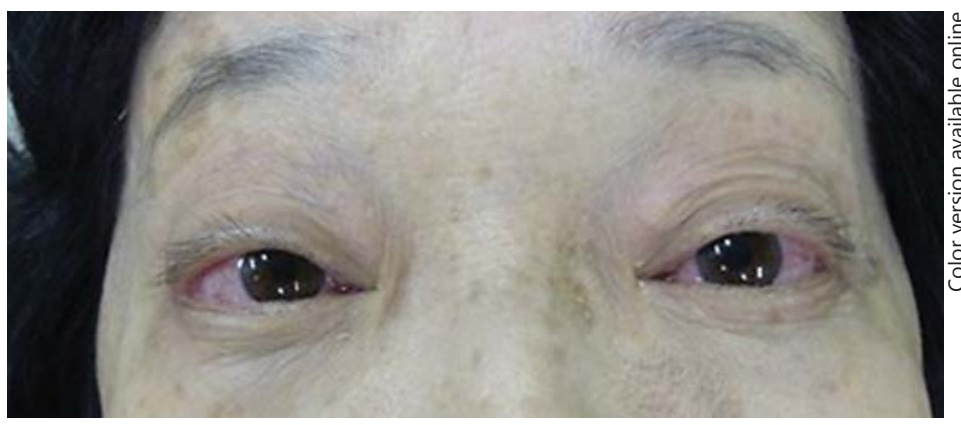

have various morphologic characteristics and angioarchitecture based on the different portions of the cavernous sinus involved [2].

We describe a patient presenting with symptoms typical of a cavernous sinus DAVF but with the DAVF actually located in the intercavernous sinus. This case is very rare, and it shows the necessity of proper localization of the fistulous site in order to successfully eliminate the disease.

\section{Case Report}

A 65-year-old right-handed woman presented with a history of double vision, pain, and redness affecting both eyes. Her symptoms had begun spontaneously before admission and gradually progressed. Physical examination showed periorbital swelling, pulsatile exophthalmos, ocular bruit, conjuctival injection and squint with associated right abducens nerve palsy (fig. 1).

Carotid angiography showed a DAVF of the intercavernous sinus fed by the meningohypophyseal branches of the right internal carotid artery and middle meningeal and distal internal maxillary branches of both external carotid arteries. The fistula drained into the superior and inferior ophthalmic veins and into the inferior petrosal sinuses (IPS). The engorged superior and inferior ophthalmic veins from retrograde venous drainage were also identified (fig. 2a).

We used both femoral veins and introduced a combination of guiding catheter and inner catheter (Envoy XB $6 \mathrm{~F} 90 \mathrm{~cm}$, Codman/Medikit 4F $107 \mathrm{~cm} \mathrm{JB} \mathrm{3)} \mathrm{and} \mathrm{navigated} \mathrm{coaxially} \mathrm{to} \mathrm{the} \mathrm{IPS.} \mathrm{Then,} \mathrm{we} \mathrm{used} \mathrm{Excelsior}$ SL-10 (Boston Scientific, Fremont, Calif., USA) and microguide wire (GT 0.012; Terumo, Tokyo, Japan); contrast medium was gently injected from the microcatheter (selective venography). In cases of an occluded IPS, a 0.035-inch guidewire was used to pass through the sinus (the rolling method) [3]. In this method, manual screw-like movements of a guidewire are employed throughout the occluded IPS to pass through it to the targeted carotid sinus. Heparin was injected after the microcatheter was in the carotid sinus. Embolization was performed with coils [Target (Stryker); ED coils (Kaneka Medix); Trufill (Orbit Galaxy); Microplex (Microvention)]. After coil placement, angiography showed no residual flow and complete obliteration of the fistula (fig. 2c, d). The patient recovered uneventfully and without complications.

\section{Discussion}

The cavernous sinus is a plexus of veins with connections to various afferent and efferent veins. The carotid cavernous fistulas involve different anatomical compartments of this space and have different drainage based on anatomical location and hemodynamic factors. In this respect, a detailed knowledge of the anatomy of this region is the mainstay in planning the most effective and targeted therapeutic approach to the lesion while preventing complications [4]. 
Melake et al.: Intercavernous Sinus Dural Arteriovenous Fistula Treated with
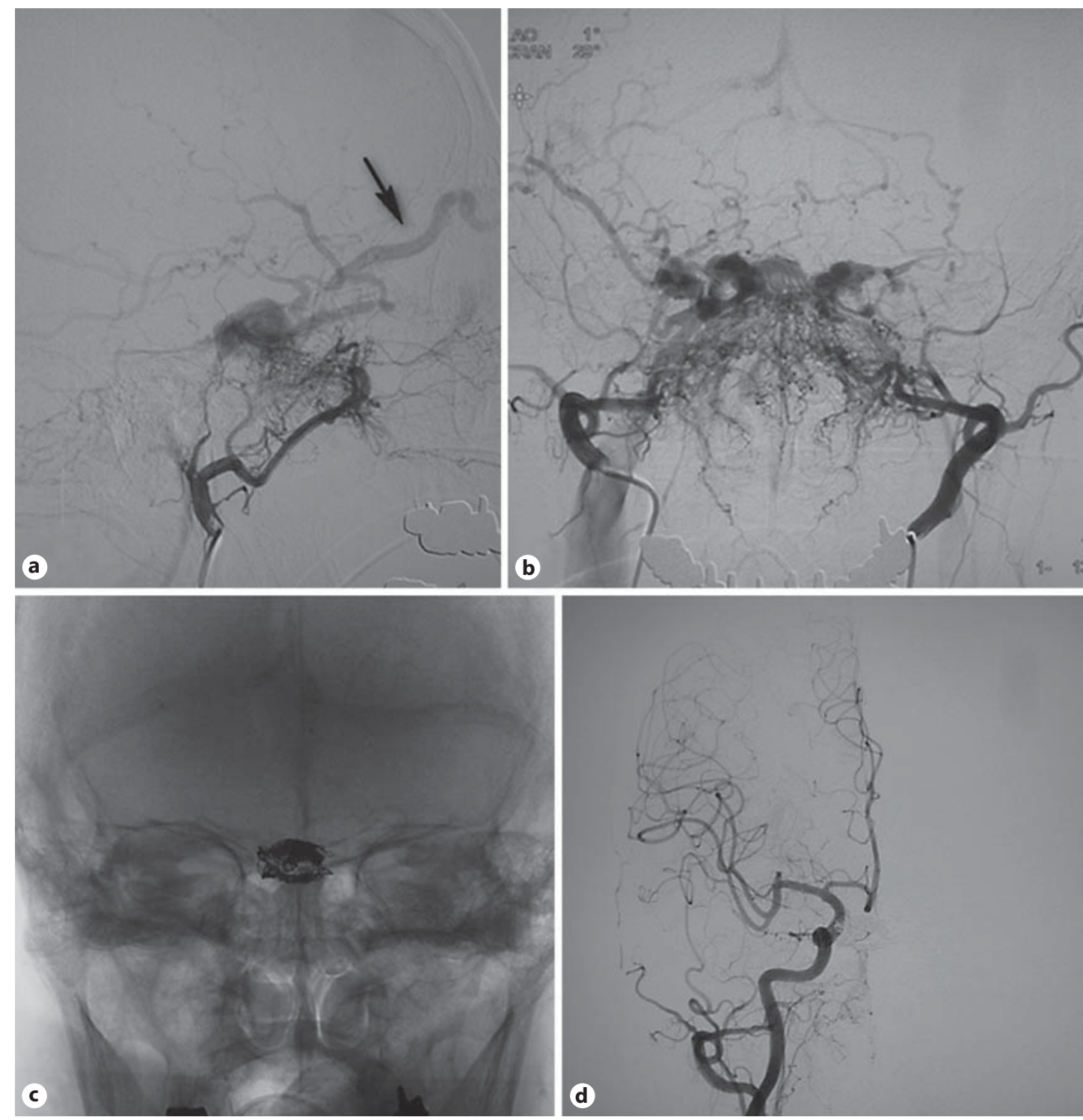

Fig. 2. Right internal maxillary selective angiography with dilated superior ophthalmic vein (arrow; a), the intercavernous DAVF (b), coils inserted in both anterior and posterior intercavernous sinuses (c), and the final image of right common carotid angiography with complete obliteration of the fistula are shown (d).

In some cases of arteriovenous fistula of the cavernous sinus, contralateral, chiefly ocular signs are observed, which implies the existence of an extensive communication between the cavernous sinuses. Such cases require a bilateral angiographic study [5]. In our case, we had bilateral ocular signs; we believe that in similar cases where bilateral ocular symptoms are so marked, the possibility of a primary lesion of the intercavernous sinuses is high and provokes the interventionist to search more deeply for an intercavernous primary site for the fistulous communication. In the present case, identification of the correct fistulous point prevented unnecessary embolization and occlusion of the surrounding normal vasculature. Such unnecessary occlusion may result in serious hemodynamic shifts. Regarding the transvenous approach, it was described by many authors to be the most applicable and the safest 
for managing cavernous and paracavernous fistulas [3]. Various transvenous approaches are available, and the choice of the preferential route depends on the type of fistula drainage. Access to the cavernous sinus through the ipsilateral IPS is usually the shortest and most favorable route because of its direct connection to the internal jugular vein through the petrooccipital fissure [2]. In cases of an occluded IPS, a 0.035-inch guidewire can be used to pass through the sinus (the rolling method) [3].

Based on the complex pattern of drainage of indirect carotid cavernous fistulas, alternative venous pathways for accessing the cavernous sinus include the contralateral IPS through the intercavernous sinus, the clival venous plexus, the cortical venous drainage, the pterygoid plexus, the inferior ophthalmic vein, and the superior ophthalmic vein through the angular or retromandibular veins. The selection of one of these alternate pathways depends on the individual characteristics of the fistula venous drainage and the anatomical position of the fistula itself in relation to the cavernous sinus. When these routes are not immediately available, percutaneous access through the superior ophthalmic vein with a surgical cutdown is a well-established alternative procedure. Direct percutaneous puncture of the cavernous sinus is also feasible and avoids the need for surgical cutdown [2]. In addition, Kim et al. [6] described a successful approach through the facial vein in cases of nonaccessible IPS cavernous and paracavernous arteriovenous fistula [7]. Our patient recovered completely without any significant complications.

\section{Conclusions}

Cases of intercavernous DAVF do exist, and they necessitate thorough neuroradiological assessment. The transvenous approach is a feasible and effective approach for complete obliteration of the fistula with an excellent outcome.

\section{References}

1 Miller NR: Carotid-cavernous sinus fistulas; in: Miller NR, Newman NJ (eds): Walsh and Hoyt's Clinical NeuroOphthalmology. Baltimore, Williams and Wilkins, 1997, pp 3263-3322.

-2 Loumiotis I, Cloft HJ, Lanzino G: Intercavernous sinus dural arteriovenous fistula successfully treated with transvenous embolization. A case report. Interv Neuroradiol 2011;17:208-211.

-3 Yoshida K, Melake M, Oishi H, Yamamoto M, Arai H: Transvenous embolization of dural carotid cavernous fistulas: a series of 44 consecutive patients. Am J Neuroradiol 2010;31:651-655.

-4 Agid R, Willinsky RA, Haw C, Souza MP, Vanek IJ, terBrugge KG: Targeted compartmental embolization of cavernous sinus dural arteriovenous fistulae using transfemoral medial and lateral facial veins approaches. Neuroradiology 2004;46:156-160.

5 Aquini MG, Marrone AC, Schneider FL: Intercavernous venous communications in the human skull base. Skull Base Surg 1994; 4:145-150.

6 Kim MJ, Shin YS, Ihn YK, Kim BM, Yoon PH, Oh SY, Kim BS: Transvenous Embolization of Cavernous and Paracavernous Dural Arteriovenous Fistula through the Facial Vein: Report of 12 Cases. Neurointervention 2013; 8:15-22.

7 Ridley H: The so-called cavernous sinus: a review of the controversy and its implications for neurosurgeons. Neurosurgery 1982;11:228-237. 